\title{
Límites y desafíos para incorporar el enfoque intercultural en la educación escolar de estudiantes inmigrantes en La Araucanía*
}

\author{
Limits and challenges to incorporate the intercultural approach \\ in school education of inmigrant students in La Araucanía
}

\author{
Héctor Torres Cuevas ${ }^{* *}$ \\ Facultad de Educación y Humanidades. Universidad del Bío-Bío, Chile. \\ htorres@ubiobio.cl
}

\begin{abstract}
RESUMEN
El artículo analiza los límites y desafíos asociados a la incorporación del enfoque intercultural en la educación escolar con estudiantes de origen inmigrante en escuelas de la Región de la Araucanía (Chile). El objetivo es discutir sobre el sentido de consolidar una educación escolar que se sustente en un enfoque intercultural, en el contexto de territorios que viven procesos de transformación cultural y demográfica por efectos de la inmigración. La estructura del trabajo considera en un primer momento el análisis de tres limitaciones asociadas con: 1) base colonial de la educación en la escuela; 2) predominio de una identidad nacional homogeneizante; y 3 ) racismo epistémico en los saberes educativos. Se finaliza con una reflexión que se enfoca en los desafíos de una educación intercultural que pongan en tensión la monoculturalidad eurocéntrica y la invisibilización de la diversidad cultural en el medio escolar.
\end{abstract}

Palabras clave: intercultural, educación escolar, inmigrante, diversidad cultural.

\begin{abstract}
This article analyzes limits and challenges associated with the incorporation of the intercultural approach in school education with immigrant origin students in elementary schools in the region of La Araucanía (Chile). The objective is to discuss the meaning of consolidating a school education that is based on an intercultural approach, in the context of territories that undergo processes of cultural and demographic transformation due to the effects of immigration. The structure of this work initially considers the analysis of three limitations associated with: 1) the colonial basis of education in the elementary school; 2) predominance of a homogenizing national identity; and 3) epistemic racism in educational knowledge. It ends with a reflection that is focused on the challenges of an intercultural education that puts tension on eurocentric monoculturalism and the invisibility of the cultural diversity in school environment.
\end{abstract}

Key words: intercultural, school education, inmigrant, cultural diversity.

\footnotetext{
* Agradecimientos y financiamientos: Proyecto Fondecyt N¹1171177 “Contextualización y territorialización de la gestión educativa de la escuela en La Araucanía" y Fondecyt $N^{\circ} 1181314$ "Diálogo de saberes educativos mapuche y escolar: construcción de una base epistémica intercultural de conocimientos".

** Departamento de Ciencias de la Educación. Facultad de Educación y Humanidades. Universidad del Bío-Bío (Chile). Correo electrónico: htorres@ubiobio.cl
} 


\section{INTRODUCCIÓN}

En este artículo abordamos límites y desafíos asociados a la incorporación del enfoque intercultural en la educación escolar de estudiantes de origen inmigrante en escuelas de la Región de La Araucanía (Chile). El objetivo es discutir sobre el sentido de consolidar una educación escolar que se sustente en un enfoque intercultural en el contexto de territorios que viven procesos de transformación cultural y demográfica por efectos de la inmigración. El contexto de análisis, siguiendo lo planteado por Akkari (2009), establece que el marco educativo de la escuela se funda en un carácter institucional monocultural, un Estadonación, una lengua de enseñanza y un currículum nacional. De esta forma, los procesos educativos se ven tensionados por un elemento fundante de la institución escolar que limita las posibilidades de una apertura educativa intercultural; situación que se ve agudizada en La Araucanía, al ser un territorio colonizado por el Estado chileno en el que se mantienen vigentes conflictos interculturales e interétnicos.

A su vez, la transformación cultural y demográfica del país está mostrando que el Estado ha sido reactivo frente al crecimiento de la inmigración, con medidas que buscan maquillar la precariedad estructural en términos de política educativa (Jiménez, Aguilera, Valdés y Hernández, 2017; Mora, 2018). Es así como estudios realizados por Tijoux (2013), Riedemann y Stefoni (2015) evidencian la presencia de actitudes racistas y xenófobas en el sistema escolar, lo que da cuenta de una violencia que vulnera la dignidad de los inmigrantes. Por eso mismo, resulta prioritario profundizar la discusión sobre la gestión de la diversidad cultural en el medio escolar, con el propósito de dar respuesta a las exigencias educativas que surgen con la inmigración contemporánea de países como Venezuela, Haití y Colombia. Más aun, en el contexto de La Araucanía en el que durante los últimos 20 años se viene desarrollando la materialización de un formato de educación intercultural focalizado en la población mapuche que ha mostrado ser insuficiente para diversificar la matriz epistémica del sistema escolar (Arias-Ortega, Quilaqueo y Quintriqueo, 2019).

La estructura del trabajo considera el siguiente orden, en un primer momento analizamos tres limitaciones asociadas con 1) base colonial de la educación en la escuela; 2) predominio de una identidad nacional homogeneizante; y 3) racismo epistémico en los saberes educativos. Se finaliza con una reflexión que se enfoca en los desafíos de una educación intercultural que pongan en tensión la monoculturalidad eurocéntrica y la invisibilización de la diversidad cultural en el medio escolar.

\section{DATOS DE CONTEXTO DE LA MIGRACIÓN EN LA ARAUCANÍA: PASADO Y PRESENTE}

\subsection{INMIGRACIÓN EUROPEA EN LA ETAPA DE INCORPORACIÓN DE LA ARAUCANÍA A LA SOBERANÍA ESTATAL CHILENA}

La inmigración en la Región de La Araucanía no es un fenómeno reciente, sino que tiene raíces históricas que han estado vinculadas con la incorporación unilateral de este territorio a la soberanía del Estado chileno durante la segunda mitad del siglo XIX (González y Bernedo, 2013). Es así como entre 1861 y 1912 se registró la llegada de colonos procedentes de Alemania, Francia, Suiza, Italia, España, Rusia y Bélgica por efecto de procesos 
intencionados por el Estado con la contratación de colonos y otros que se dieron de manera libre por inmigrantes no reclutados por agentes estatales (Zavala y Durán, 2005). Si bien, las cifras oficiales de esa época no son exactas, lo constatado por Gutiérrez (1989) permite afirmar que más de 60 mil personas arribaron a los territorios que fueron dispuestos para ser colonizados. Al respecto Zavala y Durán (2005), Zavala (2008) y Gutiérrez (1989) nos permiten constatar los siguientes elementos que caracterizaron la inmigración europea: 1) su aporte demográfico fue limitado y constituían una minoría en el territorio regional; 2) los elementos identitarios de los inmigrantes eran diversos (lengua, religión, cultura, territorio de origen) y no respondían a una identidad nacional previamente configurada; 3) la posición en sus sociedades de origen no se caracterizaba por ser privilegiada, al pertenecer a minorías regionales, grupos perseguidos (por ejemplo, en el ámbito religioso) o por experimentar una condición de vida precaria en lo económico; y 4) los inmigrantes contratados debieron responder a las exigencias adquiridas legalmente con el Estado chileno, lo que generó una carga adicional a su proceso migratorio.

En este contexto, el Estado y la elite decimonónica vieron en la inmigración europea una alternativa para consolidar los ideales de progreso y civilización eurocéntrico en un territorio en el que se debía superar desde una mirada racista la barbarie de la población mapuche (Pinto, 2003). De esta manera, el Estado diversificó la composición multiétnica y multicultural en La Araucanía en base a un doble sacrificio: 1) la voluntad de la población inmigrada de consolidar proyectos de vida que mejoraran su bienestar; y 2) la humillación y violencia colonial experimentada por la sociedad mapuche que fue confinada a un sistema de reducciones. Es así como se vivió en un primer momento histórico la inmigración en un espacio regional que se transformó desde una perspectiva económica, cultural, territorial y educativa.

\subsection{INMIGRACIÓN CONTEMPORÁNEA EN CHILE Y LA ARAUCANÍA}

Desde una perspectiva general las cifras estimadas por el Instituto Nacional de Estadísticas (INE, 2019) y el Departamento de Extranjería y Migración (DEM, 2019) indican que hasta diciembre de 2018 la población extranjera en Chile era de 1.251.225 personas. Del total de la población 646.128 son hombres y 605.097 son mujeres, estimándose que el 60\% de la población se concentra en el rango etario de 20 a 39 años. En este contexto, los 5 colectivos con mayor presencia en el país están integrados por población originaria de Venezuela (288.233 personas), Perú (223.923 personas), Haití (179.338 personas), Colombia (146.582 personas) y Bolivia (107.346 personas). Respecto a las cifras de población extranjera en La Araucanía, se constata que entre los años 2005 a 2018 se otorgaron 18.872 visas, entre las que destacan los colectivos de origen haitiano, venezolano, colombiano, argentino y peruano (13.239 personas en total). Es interesante notar que hasta el año 2017 la cantidad de visas entregadas no superaba las 2.700 por año. Sin embargo, el año 2018 se quintuplicó la cantidad de trámites y se alcanzaron las 8.454 visas otorgadas. Entonces, con los datos oficiales del DEM se observa que en un solo año la población de inmigrantes en La Araucanía creció más de un 40\%. Asimismo, la dispersión del rango etario evidencia que el 41,9\% tiene entre 18 y 29 años, un 37,7\% entre 30 y 44 años, un 9,6\% entre 45 y 59 años, un 7,9\% 17 años o menos, un 2,4\% entre 60 y 74 años y solo $0,4 \% 75$ o más años (DEM, 2019). Si bien, la cantidad de población extranjera es significativamente menor (1\%) a lo que ocurre en la Región Metropolitana que concentra más del 60\%, es importante destacar 
que territorios como La Araucanía no están quedando ajenos al fenómeno migratorio que experimenta el país, lo que está aportando a diversificar y rejuvenecer la composición de su población local.

A su vez, los datos estadísticos ponen de manifiesto que, durante los últimos años, el flujo migratorio en Chile responde principalmente a una lógica sur-sur (Elizalde, Thayer y Córdova, 2013). Es decir, dentro del contexto regional, el país se ha convertido en una plaza atractiva para encontrar mejores posibilidades de desarrollo económico, frente al aumento de las barreras migratorias promovidas por los países industrializados del norte (Mora, 2009). En este sentido, si lo vemos en una perspectiva histórica hemos transitado de una inmigración decimonónica que se caracterizó por ser de origen europeo (norte-sur), a una dinámica que involucra principalmente a personas de países de América Latina y el Caribe.

Por último, entendiendo que la inmigración está redefiniendo los espacios laborales, educativos y culturales, es de nuestro interés discutir que los marcos de referencia de la escuela no están preparados para abordar los procesos de transformación que vive la sociedad. Esto vinculado con tres aspectos: 1) la base colonial de la educación en la escuela; 2) el predominio de una identidad nacional homogeneizante; y 3 ) el racismo epistémico en los saberes educativos.

\section{LÍMITES PARA INCORPORAR UN ENFOQUE INTERCULTURAL EN LA EDUCACIÓN ESCOLAR CON POBLACIÓN INMIGRANTE}

\subsection{BASE COLONIAL DE LA EDUCACIÓN EN LA ESCUELA DE LA ARAUCANÍA}

Un primer límite que identificamos está asociado con la base colonial que tiene el origen de la escuela en La Araucanía. En este sentido, desde la segunda mitad del siglo XIX, el estado chileno utilizó la institución escolar con el propósito de asimilar a la población mapuche (Mansilla, Ponce de León y Turra, 2018). De este modo, la educación funcionó en la lógica de que los mapuches debían ser civilizados y evangelizados en la fe cristiana y era rol de la escuela aportar al cumplimiento de la labor promovida por el aparato estatal (Mansilla, Huaiquián y Pozo, 2018; Pinto, 2003). Así, lo que se impuso fue una educación basada en la idea de superioridad de los saberes, valores y lengua de la sociedad de origen europeo (etnocentrismo eurocéntrico) como una forma de establecer el control social (Ball, 1983), que exigía el proceso de ocupación de las tierras mapuches. Por eso mismo, la educación colonial constituyó un mecanismo efectivo y coherente con los propósitos estatales, por los siguientes aspectos: 1) permitir la uniformización de una sociedad; 2) instaurar sistemas de control y vigilancia; 3) establecer el orden social; y 4) facilitar la difusión de ideas políticas, religiosas y económicas (García, 2005).

Desde esta perspectiva, la educación colonial permitió sostener un proyecto educativo que despreció los saberes y lengua de la sociedad mapuche, de manera similar a lo experimentado por otros pueblos indígenas en contextos coloniales como Canadá, Nueva Zelanda, Australia o Kenia (Bull y Alia, 2004; Wane, 2008). Así, se legitimó que la escuela debía ser la responsable de transformar a los grupos de la población que debían ser colonizados, por representar una expresión de diversidad que entorpecía el desarrollo del proyecto de nación chilena. Ciertamente, obtener la sujeción de los cuerpos y el control de las poblaciones ha representado en el desarrollo de la economía capitalista la posibilidad de 
consolidar un aparato eficaz de ordenamiento de la vida (Foucault, 2002). Este ordenamiento de la vida en el marco de una educación colonial ha tenido las siguientes consecuencias: 1) imposición de un monolingüismo en la lengua del colonizador; 2) enseñanza de un currículum escolar limitado a una base monocultural eurocéntrica; 3) desprestigio social y minorización cultural de los grupos subordinados; 4) establecimiento de una educación racializada y sexista; y 5) expansión de un imperialismo cognitivo (Battiste, 2016; Sobritchea, 1989; Wane, 2008). Como analiza Wane (2008) la educación colonial erosionó las estructuras de conocimiento de las sociedades colonizadas plantando las semillas para la expansión del imperialismo. De esta forma, el sistema escolar ha impuesto una forma de hablar, teorizar y vivir en sociedades que siguen estando afectadas por prácticas coloniales que deslegitiman y marginan los conocimientos de los grupos subordinados y minorizados.

\subsection{PREDOMINIO DE UNA IDENTIDAD NACIONAL HOMOGENEIZANTE}

Un segundo elemento que limita el desarrollo de una educación intercultural coherente y pertinente con la diversificación de la población escolar se vincula a la vigencia de una concepción homogeneizante de identidad nacional en la escuela. En Latinoamérica, la construcción del Estado-nación ha tenido como elementos de referencia el dogma de la unidad republicana y el mito de la identidad nacional (Bazin, 2011). Las elites criollas después de concretada la ruptura con el modelo colonial a causa de los procesos de independencia "optaron por imponer a sus sociedades alguna variante del modelo de organización más moderno entonces disponible, el modelo republicano, representativo -y muchas veces federal-surgido de la Revolución francesa y del establecimiento de Estados Unidos como nueva nación.” (Meyer y Reyna, 1989, p. 17). Esto inició la configuración de proyectos de organización institucional en el seno de los Estados-nacionales bajo los principios de la república ${ }^{1}$ : una etapa de ensayo y error que desencadenó nuevas rupturas, acuerdos y desacuerdos que reordenaron el esquema de poder de las elites criollas, y legitimaron progresivamente un sistema político y de vida considerado moderno para las poblaciones de los territorios independizados (Salazar y Pinto, 1999; Rouquié, 2010).

En el caso chileno, la formación de la estructura política y jurídica del Estado republicano dio paso a la construcción de la nación basada en dimensiones como el orden, el patriotismo y la homogeneidad cultural de su población, con la voluntad de hacer coincidir las fronteras políticas con las fronteras culturales (Habermas, 1998; Martiniello, 2011). Entonces, un aspecto que se estimó como prioritario para la elite criolla fue la diseminación de la "chilenidad" a los grupos subalternos, utilizando con este propósito distintos medios: la iglesia, la literatura, las guerras, la educación y la prensa para transformar a los vasallos del rey en ciudadanos chilenos leales a su patria (Pinto, 2003). Esto fue configurando el sueño de la élite criolla de crear una gran familia que comparte una gran casa, una comunidad imaginada (Anderson, 2002) que se traspasa a todos y todas quienes habitan el territorio nacional, ¿con qué propósito? para erradicar todo aspecto que atentara contra la cohesión social, es decir, todas las fuerzas disfuncionales o disgregadoras: indígenas, afrodescendientes e inmigrantes.

La palabra república es la expresión de res publica lo que busca designar "la actividad pública", "los asuntos públicos", "el interés público" o "la comunidad constituida por el pueblo" (Audier, 2004, p. 7). 
De esta forma, en la sociedad se instaló la necesidad de consolidar un proyecto de orden y unidad nacional (Salazar y Pinto, 1999) a través de la aplicación de procesos de homogeneización cultural e identitario (Gallardo, 2001; Martiniello, 2011). Es así que, con el incremento del acceso a la escuela y la progresiva escolarización de la población del país (obreros, campesinos, indígenas, afrodescendientes e inmigrantes), se logró ir avanzando en la consolidación de una identidad nacional limitada a valores (heroísmo, amor a la tierra, cristianismo), símbolos (bandera y escudo nacional), héroes (Prat, O’Higgins, otros) y prácticas de corte patriota, militar, religioso y nacionalista (desfiles, actos militares, servicio militar, procesiones). Esta forma de orientar la identidad nacional ha conducido a que la ciudadanía posea altos niveles de identificación con Chile, lo que se relaciona con aspectos de lealtad a la patria, prácticas nacionales (Parada militar), eventos mediáticos (La Teletón) y estereotipos nacionales (roto chileno) (Cuevas, 2008). Si bien, no se puede hablar de una identidad nacional homogénea y fija en el tiempo, ya que sus contenidos se van ajustando a cambios históricos de la sociedad (Larraín, 2010). Retomando lo dicho por Pinto (2003) asumimos que si algo caracteriza la identidad chilena es una triple condición: 1) ser de tipo esquizofrénica; 2) basarse en la negación de la diversidad; y 3) estar en conflicto con sus elementos fundantes. Precisamente esa continua negación de la diversidad y los conflictos identitarios dan cuenta de la dificultad de diversificar los elementos que son generadores de sentido de comunidad. Este elemento genera tensión en el contexto de un sistema escolar que en territorios de La Araucanía sigue operando en la lógica de una identidad homogénea, esencializada y estática.

\subsection{RACISMO EPISTÉMICO EN LOS SABERES EDUCATIVOS}

Un tercer elemento que limita el desarrollo de la educación intercultural se refiere a la presencia de un racismo epistémico en lo saberes educativos. De acuerdo con lo propuesto por Grosfoguel (2011) entendemos que:

El racismo epistémico funciona a través de los privilegios de una política esencialista («identitaria») de las elites masculinas «occidentales», es decir, la tradición de pensamiento hegemónica de la Filosofía occidental y la teoría social que rara vez incluye a las mujeres «occidentales» y nunca incluye los/las Filósofos/as, las Filosofías y científicos/as sociales «no occidentales». En esta tradición racista/ patriarcal, se considera «Occidente» como la única tradición de pensamiento legítima capaz de producir conocimiento y como la única con acceso a la «universalidad», la «racionalidad» y la «verdad» (p. 343).

Es así que en el contexto de nuestras sociedades se ha validado una forma limitada de construcción del conocimiento, impulsada desde el siglo XVI por el proyecto colonial europeo, que ha definido a toda matriz epistémica no cristiana y no occidental como inferior y subalterna (Grosfoguel, 2016). Entonces, hemos asumido que lo digno de ser teorizado, enseñado y universalizado es la tradición de pensamiento creada eminentemente por hombres occidentales (Descartes, Kant, Hegel, Marx, Freud, entre otros), que han adquirido con sus formas de construir conocimiento una posición hegemónica en el mundo (Aguilar, 2018). Ejemplo es el legado de la filosofía cartesiana que sigue operando como referencia en el proceso de validación del conocimiento, al definir la separación sujeto-objeto, la preeminencia 
de lo escrito, la búsqueda de objetividad en el proceso de investigación y la generación de un conocimiento imparcial (Grosfoguel, 2016; Quintriqueo y Cárdenas, 2010).

De este modo, en el medio escolar la definición de los saberes educativos mantiene en sus aspectos constitutivos (teóricos y prácticos) una base epistémica eurocéntrica que excluye los saberes de los contextos indígenas, rurales, campesinos, orientales, islámicos, africanos, que entran en el rango de saberes mágicos, místicos y no científicos (Akkari, 2007; Battiste, 2016; Bodkin-Andrews y Carlson, 2016; Quintriqueo y Torres, 2012; Spivak, 2010; Wane, 2008). En este sentido, todo saber que no se ajuste de manera efectiva a los procesos de producción capitalista queda relegado a modelos secundarios, al ser considerados peligrosos para el avance de la economía de mercado (Escobar, 2010). De ahí la necesidad de minorizarlos o excluirlos por ser disruptivos con la lógica eurocentrada y judeocristiana. Es lo que ha sido demostrado por Grosfoguel (2016) con los cuatro epistemicidios practicados por las potencias coloniales europeas: 1) contra musulmanes y judíos en la conquista de Al-Andalus; 2) contra los pueblos indígenas en el continente americano y después en Asia; 3) contra los africanos aprisionados en sus territorios y después esclavizados en el continente americano; y 4) contra las mujeres que transmitían y practicaban los conocimientos indo-europeos en Europa, que fueron acusadas de brujas y quemadas vivas. Esto es lo que ha llevado a la consagración en nuestras sociedades de una matriz epistémica racista, sexista, patriarcal, colonial y capitalista.

Por lo tanto, la matriz epistémica de los saberes que son incorporados en el currículum escolar mantiene en su esencia una tendencia a reproducir la superioridad del conocimiento occidental eurocéntrico, lo que incide en el desplazamiento de las culturas, lenguas y conocimientos de los grupos de la sociedad que viven procesos de dominación o subalternidad (Akkari, 2007). Es lo que ocurre con la población escolar de origen inmigrante cuando en la sociedad de acogida vive procesos de integración (asimilación) que niegan o desconocen los marcos de conocimiento de su sociedad de origen, lo que trae por consecuencia la generación de desencuentros, fracaso escolar y vulneración de derechos (Carrasco, Pàmies y Bertrán, 2009; Joiko y Vásquez, 2016; Suárez-Orozco, Darbes, Dias y Sutin, 2011; Trueba, 1988; Trueba, Jacobs y Kirton, 2014). Entonces lo que constatamos es un nudo crítico en los sistemas escolares, al no estar preparados para construir procesos educativos que den espacio a otras matrices epistémicas, lo que conduce a que se siga perpetuando un racismo epistémico que alimenta incomprensiones y desacuerdos en el medio escolar a causa de los orígenes étnicos, culturales o lingüísticos de los estudiantes (Akkari, 2007).

\section{INTERCULTURALIZAR LA EDUCACIÓN ESCOLAR: DESAFÍOS EN EL MARCO DE LA INMIGRACIÓN CONTEMPORÁNEA}

Siguiendo lo planteado por Suárez-Orozco y Suárez-Orozco (2003), reconocemos que la escuela constituye para el estudiante inmigrante el primer lugar en el que entra en contacto de manera sistemática con la cultura de la sociedad de acogida. No obstante, en relación con las limitaciones que han sido descritas surgen dos interrogantes: 1) ¿Cómo debemos repensar la relación educativa con la población escolar migrante en contextos escolares marcados por una educación colonial, homogeneizante y racista?; y 2) ¿Qué debiéramos considerar para generar marcos de gestión de la diversidad cultural que supere la lógica que 
responsabiliza al inmigrante de su integración a la sociedad de acogida? Para el Estadonación, el rol histórico de la escuela ha sido ser la institución por excelencia encargada de transformar a los individuos en ciudadanos (Schnapper, 1994). Sin embargo, el aumento de los desplazamientos trasnacionales ha generado que el rol histórico de la escuela entre en tensión, debido a que las normas, prácticas sociales y esquemas culturales no están respondiendo de manera apropiada a la realidad de los espacios escolares (Suárez-Orozco et al., 2011). Es decir, el marco actual de nuestros sistemas escolares evidencia que tenemos una escuela desalineada con los desafíos que emergen con el aumento de la inmigración (Despagne y Jacobo-Suárez, 2016). Estos desafíos están vinculados con la necesidad de: 1) instalación en la escuela de una comprensión diversa de la identidad y la cultura: 2) construcción de espacios de inclusión que permitan desarrollar procesos de inmersión lingüística y cultural; 3) comprensión de los efectos de la migración en la experiencia de vida de los niños y sus familias; 4) reconocimiento de los factores económicos que impactan en la vida de las familias inmigrantes; 5) superación de la concepción que asume la diversidad como un problema; y 6) valoración de los conocimientos y capacidades de los estudiantes inmigrantes en una lógica contraria a la cultura del déficit (Despagne y Jacobo-Suárez, 2016; Essomba, 2012; Foley, 1997; Suárez-Orozco y Suárez-Orozco, 2003; Trueba, 2002).

Entonces, un aspecto que necesita ser discutido se refiere a cómo pensamos posicionar un enfoque educativo que asuma el desafío de la inmigración en una perspectiva que cuestione el colonialismo, el racismo y la xenofobia. En la actualidad, resulta complejo generar una propuesta educativa que revierta las asimetrías en las relaciones de poder entre los miembros de la sociedad de acogida y la población migrante. Por ejemplo, el multiculturalismo y la educación multicultural en el contexto anglosajón han mostrado ser insuficientes para resolver los conflictos entre la sociedad mayoritaria y las minorías (inmigrantes, refugiados, indígenas), ya que sus medidas han quedado limitadas a un reconocimiento y tolerancia de la diferencia, sin revertir la desigualdad en las relaciones de poder (Abdallah-Pretceille, 2004; Doytcheva, 2011; Essomba, 2006; Kymlicka, 2001). Según Tubino (2002) lo que ocurre con el multiculturalismo es que intenta radicalizar los ideales de la Ilustración sintetizados en la autonomía y la tolerancia, desarrollando una filosofía de la identidad que sea coherente con el marco contemporáneo de respeto de la diversidad cultural. De este modo, lo que se crea es un sistema de gobierno de la diferencia que permite a los Estados-nación reproducir las desigualdades internas y reafirmar su capacidad de delimitar los aspectos étnico/culturales legítimos y los que deben ser ignorados (Briones, 2002, 2005).

Por eso mismo, viendo las falencias del multiculturalismo, una posible alternativa de transformación de la educación escolar se encuentra en la educación intercultural (Essomba, 2006). Es una opción válida que necesita ser discutida, ya que en el caso de América Latina y particularmente en territorios como La Araucanía, lo que hemos experimentado es un formato funcional de educación intercultural bilingüe (EIB) que no ha logrado diversificar los procesos de enseñanza y consolidar una formación escolar antirracista y descolonizada, básicamente por tres factores: 1) focalización de la intervención educativa en la población mapuche; 2) exclusión de la intervención educativa a los grupos de la población en los que se originan el racismo y el colonialismo; y 3) perpetuación de la idea de que el indígena es el responsable de su integración a la sociedad mayoritaria (Gasché, 2010; Quilaqueo, Quintriqueo, Torres y Muñoz, 2014). 
En este sentido, los problemas asociados a la materialización de la educación intercultural necesitan ser repensados, si aspiramos a consolidar una educación escolar que responda de manera apropiada a las características de comunidades educativas con un alumnado diverso. Al respecto, un aporte lo encontramos en lo propuesto por Essomba (2006), quien señala que la escuela intercultural debe favorecer la creación de contextos socioculturales que permitan a los estudiantes aprender distintos puntos de vista culturales. De esta forma, el objetivo es consolidar el aprendizaje de competencias interculturales, para que los estudiantes puedan desenvolverse en distintos contextos de diversidad cultural a nivel local y global. Así, lo que debiera ocurrir es transitar como afirma Essomba (2006) de una escuela de código cerrado a una de código abierto, donde el conjunto de la comunidad educativa se compromete desde un plano ético y político por confrontar el etnocentrismo, la segregación y el reduccionismo de la educación monocultural y monolingüe.

Otro aporte se constata en lo planteado por Quintriqueo y Cárdenas (2010) y Quilaqueo et al. (2014) al señalar los siguientes supuestos para el desarrollo de la educación intercultural: 1) se debe asumir que en los procesos de enseñanza-aprendizaje las personas adquieren habilidades y destrezas que responden a las características de sus comunidades de origen (perspectiva intracultural); 2) se debe reconocer que lo aprendido contiene significados que favorecen la comunicación y participación de las personas en actividades propias de su grupo de origen; 3) el aprendizaje se construye desde la diferencia lo que representa una riqueza y oportunidad para la sociedad (perspectiva intercultural); 4) las prácticas pedagógicas interculturales se orientan a generar una relación dialógica entre los distintos saberes que entran en relación en el medio escolar; y 5) las relaciones entre los miembros de la comunidad educativa necesitan estar basadas en procesos de interaprendizaje, con la finalidad de propiciar procesos de construcción colaborativa del conocimiento. En consecuencia, la educación intercultural "debería tener como finalidad construir el diálogo entre sujetos que pertenecen a sociedades y culturas diferentes" (Quintriqueo, Torres, Sanhueza y Friz, 2017, p. 243). Lo anterior en la dirección de aportar a la mejora en la calidad de vida de los miembros de la sociedad que se ven confrontados en su vida cotidiana a expresiones racistas y xenófobas que atentan contra sus derechos humanos.

Por último, la propuesta teórica de Despagne y Jacobo-Suárez (2016) constituye una alternativa innovadora asociada a la creación de un tercer espacio intercultural en la escuela. Tomando como referencia lo propuesto por el intelectual de origen indio Homi Bhabha, las autoras plantean que la escuela monolítica requiere ser transformada en un espacio de interacción y co-construcción en el que se negocian y reinterpretan identidades. Esto tendría que apuntar a lo siguiente: 1) favorecer el diálogo y la co-construcción a partir de los conocimientos y experiencias de los estudiantes y sus familias; 2) potenciar la intersección entre las culturas que son vividas por los estudiantes y sus familias; y 3) conceptualizar y vivir la identidad como algo flexible y no fijo. Cada uno de los aspectos analizados conflictúa elementos estructurales de la escuela (escuela monolítica, nacionalista y racista), que han imposibilitado la valoración e inclusión de la población inmigrante desde el marco de relaciones de poder simétricas.

En síntesis, avanzar en la interculturalización del espacio escolar es un proceso que exige aperturas epistémicas, políticas y culturales que no están instaladas en la actualidad en el sistema escolar. Al contrario, se carece de una posición crítica en actores claves del sistema escolar (ministerio, sostenedores, directivos, profesores, formadores de docentes) 
que pongan en tensión el legado de la educación colonial, homogeneizante y racista. Al respecto, la educación intercultural puede ser un aporte para la gestión de la diversidad cultural en territorios fragmentados por diferencias culturales y étnicas. Sin embargo, se requiere mayor debate académico, social, político y curricular, para evitar la reproducción de procesos que han mostrado el predominio de la indiferencia por las diferencias (Bourdieu, 1966). Es lo que podemos aprender de lo experimentado durante fines del siglo XIX y las dos primeras décadas del siglo XX en La Araucanía, con una educación escolar que finalmente erradicó la diversidad de identidades y lenguas de la población migrante (suizos, italianos, franceses, alemanes, ingleses, árabes), imponiendo una lengua, cultura e identidad nacional. Si bien, en la actualidad los datos estadísticos muestran que, en La Araucanía, el total de población migrante es un porcentaje mínimo del total de la población regional; esto no puede ser un factor autocomplaciente que limite la urgencia de reflexionar y generar las transformaciones que exige el sistema escolar. Para cerrar una evidencia curricular, en primero básico la cuarta unidad de la asignatura de Historia, Geografía y Ciencias Sociales, busca que los niños "reconozcan símbolos y expresiones de la identidad nacional y de las diversas identidades locales" (Ministerio de Educación [MINEDUC], 2012, p. 121). Con esto lo que se espera es:

que se reconozcan como chilenos y chilenas que habitan en un país con características propias, se motiven por conocerlo más y valoren su identidad nacional. Al mismo tiempo, se busca que conozcan tradiciones de su localidad, región y país, fortaleciendo el sentido de pertenencia regional y nacional (p. 121).

A pesar de que en la introducción del programa se indica que el profesor/a debe atender la diversidad de sus estudiantes, en la definición curricular lo que constatamos es una propuesta que sigue anclada a una formación nacionalista y homogeneizante. De ahí que, la discusión sobre la necesidad de consolidar un enfoque intercultural de la educación se mantenga como un desafío a construir en nuestros territorios.

\section{CONCLUSIONES}

En conclusión, la base colonial de la educación escolar, la formación de una identidad nacional homogeneizante y el racismo epistémico en los saberes educativos son limitaciones que, si siguen estando vigentes, difícilmente abrirán opciones para la interculturalización de la educación escolar. Al respecto, se debe ir más allá del simple reconocimiento de la heterogeneidad cultural de los estudiantes, para transitar a propuestas concretas que pongan en tensión los nudos críticos de un sistema escolar anclado a un modelo monocultural y monolingüe de base eurocéntrica. Esto exige contar con métodos, competencias y enfoques pedagógicos capaces de facilitar la inserción social, educativa, cultural, lingüística y epistémica de los estudiantes inmigrantes. De esta forma, se podría generar transformaciones a los marcos culturales y epistémicos de la educación escolar para transitar a la definición de una matriz inter-cultural e inter-epistémica, que logre articular distintas visiones de mundo, historias, identidades, saberes y lenguas. 


\section{REFERENCIAS BIBLIOGRÁFICAS}

Abdallah-Pretceille, M. (2004). L'éducation interculturelle. París: Presses universitaires de France.

Aguilar, E. (2018). Rastreando el origen de las estructuras del conocimiento occidental fundadas en el racismo epistémico. Hacia una nueva propuesta para la descolonización del pensamiento. Praxis: Revista del departamento de filosofía, (77), 1-23.

Akkari, A. (2007). Les impasses de l'école multiculturelle et de l'école républicaine dans une perspective comparative. Schweizerische Zeitschrift für Bildungswissenschaften, 29(3), 379398.

(2009). Introduction aux approches interculturelles en éducation. Genève: Université de Genève.

Anderson, B. (2002). L'imaginaire national. Réflexions sur l'origine et l'essor du nationalisme. Paris: La Découverte.

Arias-Ortega, K., Quilaqueo, D. y Quintriqueo, S. (2019). Intercultural bilingual education in La Araucanía: main epistemological limitations. Educação e Pesquisa, 45, 1-16.

Audier S. (2004). Les théories de la république. París: La decouverte.

Ball, S. (1983). Imperialism, social control and the colonial curriculum in Africa. Journal of Curriculum Studies, 15(3), 237-263.

Battiste, M. (2016). Living Treaties. Narrating Mi'kmaw Treaty Relations. Halifax: Cape Breton University Press.

Bazin L. (2011). L'Etat et l'interculturalité : Le dernier bastion du colonialisme? En A. Lavanchy, A. Gajardo \& F. Dervin (Eds.), Anthropologies de l'interculturalité (pp. 101-125). París: L'Harmattan.

Bodkin-Andrews, G. \& Carlson, B. (2016). The legacy of racism and Indigenous Australian identity within education. Race Ethnicity and Education, 19(4), 784-807.

Bourdieu, P. (1966). L'école conservatrice: Les inégalités devant l'école et devant la culture. Revue française de sociologie, 7(3), 325-347.

Briones, C. (2002). Viviendo a la sombra de naciones sin sombra: poéticas y políticas de (auto) marcación de 'lo indígena' en las disputas contemporáneas por el derecho a una educación intercultural. En N. Fuller (Ed.), Interculturalidad y Política: desafíos y posibilidades. Red para el Desarrollo de las Ciencias Sociales en Perú (pp. 381-417). Lima: PUCP-UP-IEP.

. (2005). Formaciones de alteridad: contextos globales, procesos nacionales y provinciales. En C. Briones (Comp.), Cartografías argentinas (pp. 9-36). Buenos Aires: Antropofagia.

Carrasco, S., Pàmies, J. y Bertrán, M. (2009). Familias inmigrantes y escuela: Desencuentros, estrategias y capital social1. Revista complutense de educación, 20(1), 55-78.

Cuevas, H. (2008). La cuestión de la identidad chilena. Percepciones y actitudes sociales, $4^{\circ}$ Informe de la Encuesta Nacional ICSO-UDP. Instituto de Investigación en Ciencias Sociales, Chile, 133144.

Bull, S. y Alia, V. (2004). Unequaled Acts of Injustice: Pan $\square$ Indigenous Encounters with Colonial School Systems. Contemporary Justice Review, 7(2), 171-182.

Departamento de Extranjería y Migración (2019). Estadísticas Migratorias. Santiago: Gobierno de Chile.

Despagne, C. y Jacobo-Suárez, M. (2016). Desafíos actuales de la escuela monolítica mexicana: el caso de los alumnos migrantes transnacionales. Sinéctica, (47), 1-17.

Doytcheva, M. (2011). Le multiculturalisme. Paris: La Découverte.

Elizalde, A., Thayer, L. y Córdova, M. (2013). Migraciones sur-sur: paradojas globales y promesas locales. Polis. Revista Latinoamericana, 12(35), 7-13.

Escobar, A. (2010). Territorios de diferencia: lugar, movimientos, vida, redes. Popayán: Envión Editores.

Essomba, M. À. (2006). Liderar escuelas interculturales e inclusivas: equipos directivos y 
Estudios Pedagógicos XLV, $\mathrm{N}^{\circ}$ 1: 155-167, 2019

LÍMITES Y DESAFÍOS PARA INCORPORAR EL ENFOQUE INTERCULTURAL EN LA EDUCACIÓN ESCOLAR DE

ESTUDIANTES INMIGRANTES EN LA ARAUCANÍA

profesorado ante la diversidad cultural y la inmigración. Barcelona: Graó.

. (2012). Inmigración, sociedad y educación en la UE. Hacia una política educativa de plena inclusión. Cultura y Educación, 24(2), 137-148.

Foley, D. (1997). Deficit thinking models based on culture: The anthropological protest. En R. Valencia (Ed.), The evolution of deficit thinking: Educational thought and practice (pp. 113131). Washington, DC \& London: Falmer.

Foucault, M. (2002). Vigilar y castigar: nacimiento de la prisión. Buenos Aires: Siglo XXI Editores Argentina.

Gallardo, V. (2001). Héroes indómitos, bárbaros y ciudadanos chilenos: el discurso sobre el indio en la construcción de la identidad nacional. Revista de historia indígena, (5), 119-134.

García, B. (2005). La educación colonial en la Nueva Granada: entre lo doméstico y lo público. Revista Historia de la Educación Latinoamericana, (7), 217-238.

Gasché J. (2010). De hablar de la educación intercultural a hacerla. Mundo Amazónico, 1,111-134.

González, J. y Bernedo, P. (2013). Cartografía de la transformación de un territorio: La Araucanía 1852-1887. Revista de Geografía Norte Grande, (54), 179-198.

Grosfoguel, R. (2011). Racismo epistémico, islamofobia epistémica y ciencias sociales coloniales. Tabula Rasa, (14), 341-355.

(2016). A estrutura do conhecimento nas universidades ocidentalizadas: racismo/sexismo epistêmico e os quatro genocídios/epistemicídios do longo século XVI. Sociedade e Estado, $31(1), 25-49$.

Gutiérrez, H. (1989). La inmigración española, italiana y portuguesa: Chile 1860-1930. Notas de Población, (48), 61-79.

Habermas, J. (1998). L'intégration républicaine: essais de théorie politique. París: Fayard.

Instituto Nacional de Estadísticas y el Departamento de Extranjería y Migración. (2019). Estimación de Personas Extranjeras Residentes en Chile 31 de diciembre 2018. Santiago: Gobierno de Chile.

Jiménez, F., Aguilera, M., Valdés, R. y Hernández, M. (2017). Migración y escuela: Análisis documental en torno a la incorporación de inmigrantes al sistema educativo chileno. Psicoperspectivas, 16(1), 105-116.

Joiko, S. y Vásquez, A. (2016). Acceso y elección escolar de familias migrantes en Chile: No tuve problemas porque la escuela es abierta, porque acepta muchas nacionalidades. Calidad en la Educación, (45), 132-173.

Kymlicka, W. (2001). La citoyennetté multiculturelle. Une théorie libérale du droit des minorités. Boréal: Montréal.

Mansilla, J., Huaiquián, C. y Pozo, G. (2018). Infancia mapuche encerrada: internados de las escuelasmisiones en la Araucanía, Chile (1900-1935). Revista Brasileira de Educação, 23, 1-28.

Mansilla, J., Ponce de León, M. y Turra, O. (2018). Entre la voluntad intercultural y la voluntad de asimilación: el valor de la lengua mapuche. Misioneros capuchinos bávaros y mapuche en la Araucanía 1896-1929. Opción, 34(87), 20-49.

Martiniello, M. (2011). La démocratie multiculturelle : citoyenneté, diversité, justice sociale. París: Presses de Sciences.

Meyer, L. y Reyna, J. L. (Eds.) (1989). Los sistemas políticos en América Latina. México: Siglo XXI.

Ministerio de Educación [MINEDUC] (2012). Historia, Geografía y Ciencias Sociales Programa de Estudio Primer Año Básico. Santiago: Gobierno de Chile.

Mora, C. (2009). Estratificación social y migración intrarregional: algunas caracterizaciones de la experiencia migratoria en Latinoamérica. Universum, 24(1), 128-143.

Mora, M. (2018). Política educativa para migrantes en Chile: un silencio elocuente. Polis (Santiago), 17(49), 231-257.

Larraín, J. (2010). Identidad chilena y el bicentenario. Estudios públicos (Santiago), (120), 5-30.

Pinto, J. (2003). La formación del Estado y la nación, y el pueblo mapuche. De la inclusión a la 
exclusión. Santiago: Universidad de Santiago.

Quilaqueo, D., Quintriqueo, S., Torres, H. y Muñoz, G. (2014). Saberes educativos mapuches: aportes epistémicos para un enfoque de educación intercultural. Chungará, 46(2), 271-284.

Quintriqueo, S. y Cárdenas, P. (2010). Educación intercultural en contexto mapuche: hacia una articulación entre conocimiento mapuche y conocimiento disciplinario en ciencia. En D. Quilaqueo, S. Quintriqueo y C. Fernández (Eds.), Interculturalidad en contexto mapuche (pp. 89-126). Neuquén: Editorial de la Universidad Nacional del Comahue.

Quintriqueo, S. y Torres, H. (2012). Distancia entre el conocimiento mapuche y el conocimiento escolar en contexto mapuche. Revista electrónica de investigación educativa, 14(1), 16-33.

Quintriqueo, S., Torres, H., Sanhueza, S. y Friz, M. (2017). Competencia comunicativa intercultural: formación de profesores en el contexto poscolonial chileno. Alpha, (45), 235-254.

Riedemann, A. y Stefoni, C. (2015). Sobre el racismo, su negación, y las consecuencias para una educación anti-racista en la enseñanza secundaria chilena. Polis. Revista Latinoamericana, 14(42), 191-216.

Rouquié, A. (2010). A l'ombre des dictatures. La démocratie en Amérique latine. París: Albin Michel.

Salazar G. y Pinto, J. (1999). Historia contemporánea de Chile: Actores, identidad y movimiento. Santiago: Lom Ediciones.

Schnapper, D. (1994). La communauté des citoyens. París: Gallimard.

Sobritchea, C. (1989). American colonial education and its impact on the status of Filipino women. Asian Studies, 27, 70-91.

Spivak, G. (2010). Crítica de la razón poscolonial: hacia una crítica del presente evanescente. España: Ediciones Akal.

Suárez-Orozco, M., Darbes, T., Dias, S. \& Sutin, M. (2011). Migrations and schooling. Annual Review of Anthropology, 40, 311-328.

Suárez-Orozco, C. y Suárez-Orozco, M. (2003). La infancia de la inmigración. Madrid: Ediciones Morata.

Tijoux, M. (2013). Las escuelas de la inmigración en la ciudad de Santiago: Elementos para una educación contra el racismo. Polis. Revista Latinoamericana, 12(35), 287-307.

Trueba, H. (1988). Culturally based explanations of minority students' academic achievement. Anthropology \& Education Quarterly, 19(3), 270-287.

Trueba, H. (2002). Multiple ethnic, racial, and cultural identities in action: From marginality to a new cultural capital in modern society. Journal of Latinos and education, 1(1), 7-28.

Trueba, H., Jacobs, L. y Kirton, E. (2014). Cultural Conflict \& Adaptation. Estados Unidos: Routledge.

Tubino, F. (2002). Entre el multiculturalismo y la interculturalidad: más allá de la discriminación positiva. En N. Fuller (Ed.), Interculturalidad y Política: desafíos y posibilidades. Red para el Desarrollo de las Ciencias Sociales en Perú (pp. 51-76). Lima: PUCP-UP-IEP.

Wane, N. (2008). Mapping the field of indigenous knowledges in anti $\square$ colonial discourse: A transformative journey in education. Race Ethnicity and Education, 11(2), 183-197.

Zavala, J. (2008). Los colonos y la escuela en la Araucanía: Los inmigrantes europeos y el surgimiento de la educación privada laica y protestante en la región de la Araucanía (1887-1915). Universum, 23(1), 268-286.

Zavala, J. y Durán, T. (2005). Flujos migratorios e identidades culturales en La Araucanía: reflexiones desde un acercamiento histórico-antropológico de los desplazamientos humanos. CUHSO. Cultura-Hombre-Sociedad, 10(2), 37-49. 
